\title{
THE DOW JONES INDUSTRIAL AVERAGE (DJIA) STOCK MARKET INDEX AND THE CHAOTIC GROWTH MODEL
}

\author{
Vesna Jablanovic ${ }^{1}$ (iD \\ DOI: https://doi.org/10.31410/ITEMA.2020.113
}

\begin{abstract}
The Dow Jones Industrial Average (DJIA) index includes the stocks of 30 of the largest companies in the United States. It represents about a quarter of the value of the entire U.S. stock market. The changes in the DJIA index are often considered to be representative of the entire stock market. The basic aims of this paper are: firstly, to create the simple chaotic the DJIA stock market index growth model that is capable of generating stable equilibria, cycles, or chaos; secondly, to analyze the local stability of the DJIA index movements in the period 1982-2009; and thirdly, to discover the equilibrium level of the DJIA index in the observed period. This paper confirms the existence of the stable convergent fluctuations of the DJIA index in the observed period. Also, the golden ratio can be used to define the equilibrium level of the DJIA index in the presented chaotic model.
\end{abstract}

Keywords: DJIA index, Stability, Elliot waves, Chaos.

\section{INTRODUCTION}

7 he Dow Jones Industrial Average (DJIA) includes the stocks of 30 of the largest companies in the United States. It is a price-weighted index. It represents about a quarter of the value of the entire U.S. stock market. The changes in the index explain the entire stock market dynamics. The Dow Jones Industrial Average (DJIA) was started on May 26, 1896 by Charles Dow, the editor of the Wall Street Journal and co-founder of Dow Jones \& Co. On May 26, 1896, the Dow Jones industrial average began measuring stocks (12). Further, in 1928, the sum of the prices of the 30 stocks was divided by a special number called the "divisor" rather than by 30 . This index was still identified as an "average." The Dow Jones Industrial Average (DJIA) is now based on the prices of the stocks of 30 major U.S. companies. The Dow Jones Industrial Average (DJIA) has following characteristics: (i) each company in the index is weighted by the price of its stock; (ii) the companies in the index are not representative of the market as a whole; (iii) the DJIA excludes dividend distributions; and (iv) the DJIA has evolved to include large tech and financial-services players.

Chaos theory started with Lorenz's (1963) discovery of complex dynamics arising from three nonlinear differential equations leading to turbulence in the weather system. Li and Yorke (1975) discovered that the simple logistic curve can exhibit very complex behavior. Further, May (1976) described chaos in population biology. Chaos theory has been applied in economics by Benhabib and Day (1981,1982), Day (1982, 1983), Grandmont (1985), Goodwin (1990), Medio (1993), Lorenz (1993), Jablanovic (2011, 2012, 2013, 2016), Puu, T. (2003), Zhang W.B. (2006), etc.

1 University of Belgrade, Faculty of Agriculture, Nemanjina 6, 11081 Belgrade, Serbia 
Figure 1. Interactive chart of the Dow Jones Industrial Average (DJIA) stock market index for the last 100 years. Historical data is inflation-adjusted using the headline CPI and each data point represents the month-end closing value. The current month is updated on an hourly basis with today's latest value. The current price of the Dow Jones Industrial Average as of July 02, 2020 is 25,827.36.

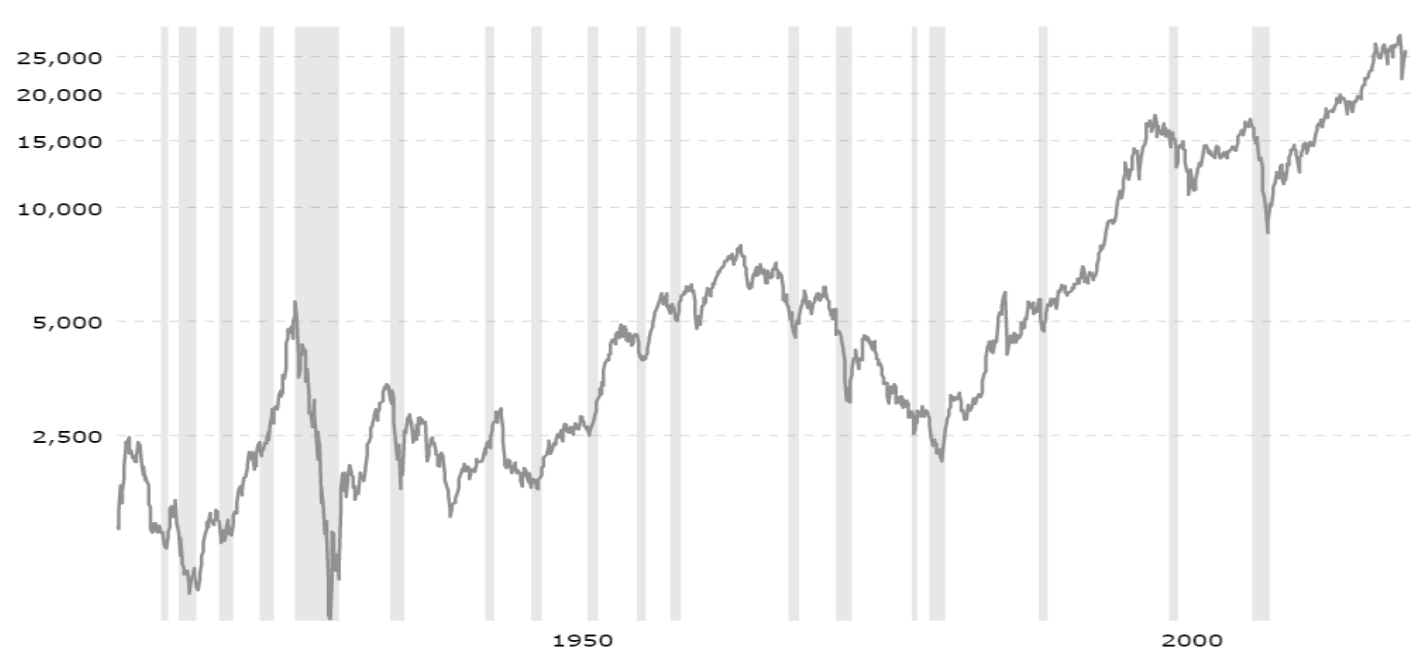

Source: http://www.macrotrends.net/1319/dow-jones-100-year-historical-chart

\section{THE MODEL}

The chaotic stock price and /or the Dow Jones Industrial Average (DJIA) growth model is presented by the following equations:

$\mathrm{D}_{\mathrm{t}}=\alpha-\beta \mathrm{P}_{\mathrm{t}} \quad \alpha>0, \beta>0$

$\mathrm{S}_{\mathrm{t}}=\gamma \mathrm{P}_{\mathrm{t}} \quad \gamma>0$

$\frac{P_{t+1}-P_{t}}{P_{t}}=\mu\left(D_{t}-S_{t}\right) \quad \quad \mu>0$

Where $\mathrm{P}_{\mathrm{t}}-$ stock price; $\mathrm{S}_{\mathrm{t}}$ - supply function of stock; $\mathrm{D}_{\mathrm{t}}$ - demand function for stock; $\mu$ the adjustment coefficient; $\alpha, \beta$ - the coefficients of the stock demand function; $\gamma$ - the coefficient of the stock supply function.

(1) defines demand function for stock; (2) defines supply function of stock; (3) determines the relation between stock price growth rate and surplus of demand for stock.

By substitution one derives:

$\mathrm{P}_{\mathrm{t}+1}=(1+\mu \alpha) \mathrm{P}_{\mathrm{t}}-\mu(\beta+\gamma) \mathrm{P}_{\mathrm{t}}^{2} \quad \alpha, \beta, \mu, \gamma>0$

Further, it is assumed that the current value of the stock price is restricted by its maximal value in its time series. This premise requires a modification of the growth law. Now, the stock price growth rate depends on the current size of the stock price, $\mathrm{P}$, relative to its maximal size in its time series $\mathrm{P}^{\mathrm{m}}$. We introduce $\mathrm{s}$ as $\mathrm{s}=\mathrm{P} / \mathrm{P}^{\mathrm{m}}$. Thus $\mathrm{s}$ range between 0 and 1 . Again we index $\mathrm{s}$ by 
$\mathrm{t}$, i.e. write $\mathrm{s}_{\mathrm{t}}$ to refer to the size at time steps $\mathrm{t}=0,1,2,3, \ldots$ Now growth rate of the stock price is measured as

$$
\mathrm{S}_{\mathrm{t}+1}=(1+\mu \alpha) \mathrm{s}_{\mathrm{t}}-\mu(\beta+\gamma) \mathrm{st}^{2} \quad \alpha, \beta, \mu, \gamma>0
$$

This model given by equation (5) is called the logistic model. For most choices of $\alpha, \beta, \gamma$, and $\mu$ there is no explicit solution for (5). This is at the heart of the presence of chaos in deterministic feedback processes. Sensitive dependence on initial conditions is one of the central ingredients of what is called deterministic chaos.

The logistic map is often cited as an example of how complex, chaotic behavior can arise from very simple non-linear dynamical equations. The map was popularized by the biologist Robert May (1976). The logistic model was originally introduced as a demographic model by Pierre François Verhulst.

It is possible to show that iteration process for the logistic equation

$$
\mathrm{z}_{\mathrm{t}+1}=\pi \mathrm{z}_{\mathrm{t}}\left(1-\mathrm{z}_{\mathrm{t}}\right), \quad \pi \in[0,4] \quad, \quad \mathrm{z}_{\mathrm{t}} \in[0,1]
$$

is equivalent to the iteration of growth model (5) when we use the identification

$$
z_{t}=\frac{\mu(\beta+\gamma)}{(1+\mu \alpha)} p_{t} \quad \text { and } \quad \pi=(1+\mu \alpha)
$$

Using (5.) and (7.) we obtain:

$$
\begin{gathered}
z_{t+1}=\frac{\mu(\beta+\gamma)}{(1+\mu \alpha)} p_{t+1}=\frac{\mu(\beta+\gamma)}{(1+\mu \alpha)}\left\{(1+\mu \alpha) p_{t}-\mu(\beta+\gamma) p_{t}^{2}\right\}= \\
=\mu(\beta+\gamma) p_{t}-\left[\frac{\mu^{2}(\beta+\gamma)^{2}}{(1+\mu \alpha)}\right] p_{t}{ }^{2}
\end{gathered}
$$

On the other hand, using (6.) and (7.) we obtain:

$$
\begin{aligned}
& z_{t+1}=\pi z_{t}\left(1-z_{t}\right)=(1+\mu \alpha)\left[\frac{\mu(\beta+\gamma)}{(1+\mu \alpha)}\right] p_{t}\left\{1-\left[\frac{\mu(\beta+\gamma)}{(1+\mu \alpha)}\right] p_{t}\right\}= \\
& =\mu(\beta+\gamma) p_{t}-\left[\frac{\mu^{2}(\beta+\gamma)^{2}}{(1+\mu \alpha)}\right] p_{t}{ }^{2}
\end{aligned}
$$


Thus, we have that iterating (5) is really the same as iterating (6) using (7). It is important because the dynamic properties of the logistic equation (6) have been widely analyzed (Li and Yorke (1975), May (1976)).

It is obtained that: (i) For parameter values $0<\pi<1$ all solutions will converge to $\mathrm{z}=0$; (ii) For $1<\pi<3,57$ there exist fixed points the number of which depends on $\mu$; (iii) For $1<\pi<$ 2 all solutions monotonically increase to $\mathrm{z}=(\pi-1) / \pi$; (iv) For $2<\pi<3$ fluctuations will converge to $\mathrm{z}=(\pi-1) / \pi$; (v) For $3<\pi<4$ all solutions will continuously fluctuate; (vi) For $3,57<\pi<4$ the solution become "chaotic" which means that there exist totally aperiodic solution or periodic solutions with a very large, complicated period. This means that the path of $\mathrm{z}_{\mathrm{t}}$ fluctuates in an apparently random fashion over time, not settling down into any regular pattern whatsoever. Also, for $\pi=2.6178$ then fluctuations will converge to $\mathrm{z}=0.618$.

The Fibonacci sequence, starting with zero and one, is created by adding the previous two numbers $(0,1,1,2,3,5,8,13,21,34,55,89,144,233,377, \ldots)$. This sequence is significant because of the golden ratio. The ratio of any number in the Fibonacci sequence relative to the number directly to its right is approximately 0.618 . The Golden Ratio (golden mean, golden number, golden proportion) is 0.618 . Adding the number 1 to the Golden Ratio $=$ Phi $(\Phi$ $=1.618$ ). Both 0.618 and 1.618 are used interchangeably to represent the golden ratio because they represent the same geometric relationship. (Lidwell, Holden, and Butler, 2010, p.114).

\section{EMPIRICAL EVIDENCE}

The main aim of this analysis is to present stock market index (DJIA) growth stability in the period 1982-2009, by using the presented non-linear, logistic model (8) because stock market index is computed from the prices of selected stocks.

In this sense,

$$
\mathrm{s}_{\mathrm{t}+1}=\omega \mathrm{s}_{\mathrm{t}}-\mathrm{v} \mathrm{s}_{\mathrm{t}}^{2} \quad \omega, \mathrm{v}>0
$$

where s - stock market index, $\omega=\pi=(1+\mu \alpha)$ and $v=\mu(\beta+\gamma)$

Firstly, we transform data on stock market index from 0 to 1 , according to our supposition that actual value of stock market index, $\mathrm{P}$, is restricted by its highest value in the time-series, $\mathrm{P}^{\mathrm{m}}$. Further, we obtain time-series of $\mathrm{s}=\mathrm{P} / \mathrm{P}^{\mathrm{m}}$. Now, we estimate the model (8). Secondly, data on the Dow Jones Industrial Average (DJIA) are transformed (Source: http://www.macrotrends.net/1319/dow-jones-100-year-historical-chart) from 0 to 1, according to our supposition that actual values of DJIA, $\mathrm{P}$, is restricted by its highest value in the timeseries, $\mathrm{P}^{\mathrm{m}}$. Further, we obtain time-series of $\mathrm{s}=\mathrm{P} / \mathrm{P}^{\mathrm{m}}$ (see Table 1). Also, the Fibonacci ratios are associated to Elliott wave pattern.

It is supposed that the basic Elliott wave pattern exists in the period Jun 1982-March 2009. This wave pattern consists of an impulse wave and a corrective wave. Impulse waves consist of 5 waves and moves in the direction of the trend. Waves 1, 3, and 5 moves in the direction of the trend, while Waves 2 and 4 move opposite to the trend. Corrective waves can be simple or complex. A simple correction consists of 3 waves (Wave A, B and C) which retrace a portion of impulse. The Elliot Wave Theory identifies (Frost A.J. \& R.P. Prechter, 2006): (i) impulse waves that set up a pattern; and (ii) corrective waves that oppose the larger trend. The stock price movements are divided into: (i) trends (five waves in the direction of the main trend); and (ii) corrections (three corrective waves) (see Fig.2.and Fig.3). 
The model (8) is estimated. The results are presented in Table 2.

Table 1. Data on the Dow Jones Industrial Average (DJIA) are transformed and Fibonacci ratios

\begin{tabular}{|r|r|r|r|r|r|}
\hline & & DJIA & P/P & Fibonacci & ratios \\
\hline & Jun-82 & 2145.93 & 0.122554 & 0.125 & $1 / 8$ \\
\hline $\mathbf{1}$ & Sep-87 & 5789.7 & 0.330649 & 0.333 & $1 / 3$ \\
\hline $\mathbf{2}$ & Nov-87 & 4074.15 & 0.232674 & 0.231 & $3 / 13$ \\
\hline $\mathbf{3}$ & May-98 & 14017.42 & 0.800533 & 0.8 & $2 / 5+2 / 5$ \\
\hline $\mathbf{4}$ & Aug-98 & 11828.80 & 0.675541 & 0.663 & $2 / 3$ \\
\hline $\mathbf{5}$ & Dec-99 & 17510.11 & 1 & 1 & $1 / 1$ \\
\hline $\mathbf{A}$ & Sep-02 & 10757.76 & 0.614374 & 0.618 & $34 / 55$ \\
\hline B & Sep-07 & 17091.62 & 0.9761 & 0.993 & $34 / 55+3 / 8$ \\
\hline C & Mar-09 & 9168.75 & 0.523626 & 0.5 & $1 / 2$ \\
\hline
\end{tabular}

Source: http://www.macrotrends.net/1319/dow-jones-100-year-historical-chart

Table 2. The estimated model (8): DJIA, Jun 1982-March 2009.

\begin{tabular}{|l|c|c|}
\hline $\mathrm{R}=0.63435$ Variance explained $40.240 \%$ & $\mathrm{v}$ \\
\hline $\mathrm{N}=8$ & $\omega(\pi)$ & 2.165967 \\
\hline Estimate & $\mathbf{2 . 7 2 2 2 8 7}$ & 0.641759 \\
\hline Std.Err. & 0.553709 & 3.375047 \\
\hline $\mathrm{t}(6)$ & 4.916454 & 0.014949 \\
\hline p-level & 0.002606 & \\
\hline
\end{tabular}

From Jun 1982 to March 2009, the Dow Jones Industrial Average moved from 2145,93 to 9168,75 . As we can see the pattern exactly follows the wave pattern described by Elliot Wave Theory. According to the logistic equation, for $2<\pi<3$ fluctuations converge to $\mathrm{z}=(\pi-1) /$ $\pi$, or (2.722287-1)/2.722287=0.63266. According to (7.), the equilibrium value of the Dow Jones Industrial Average was $0.63266 /(2.165967 / 2.722287)$, or $0.63266 / 0.795642$ or 0.795157 in the observed period. The equilibrium value of the Dow Jones Industrial Average was $0.795157 \times 17510.11=13923.287$ in the observed period.

Figure 2. The Elliott wave pattern consists of an impulse wave and a corrective wave, DJIA, Jun 1982-March 2009.

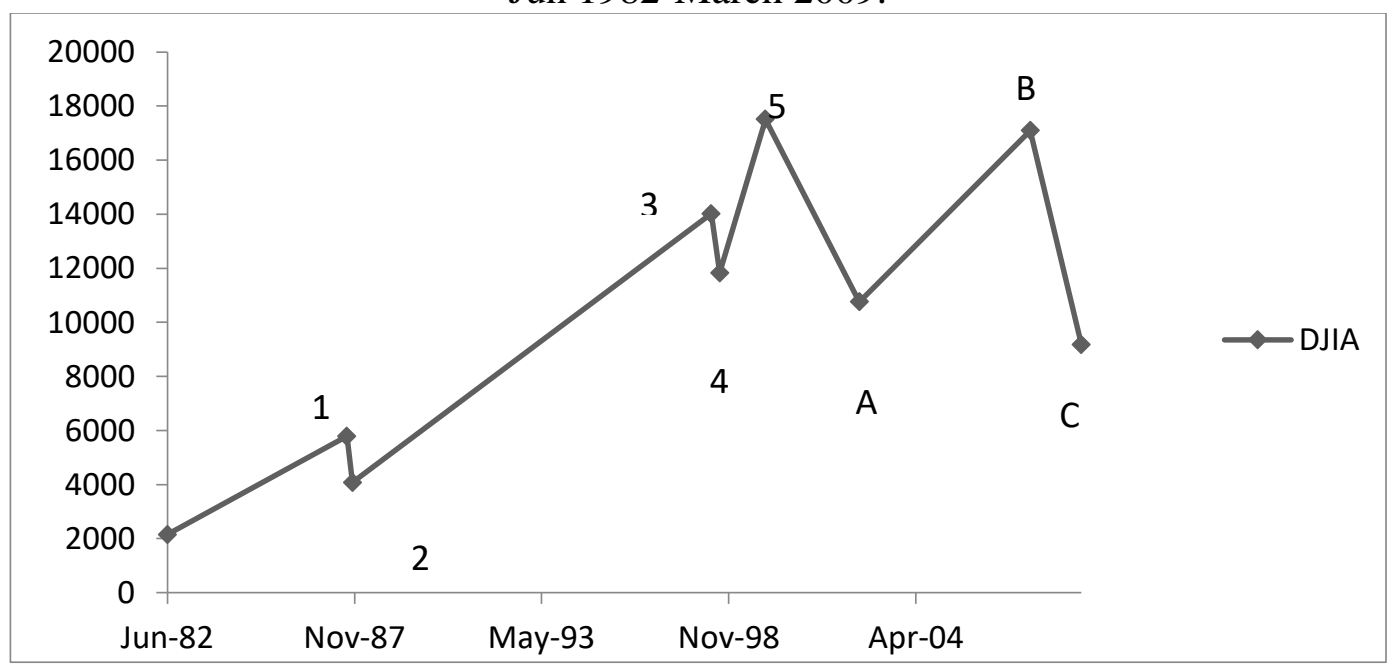


Figure 3. The Elliott wave pattern, Jun 1982- March 2009.

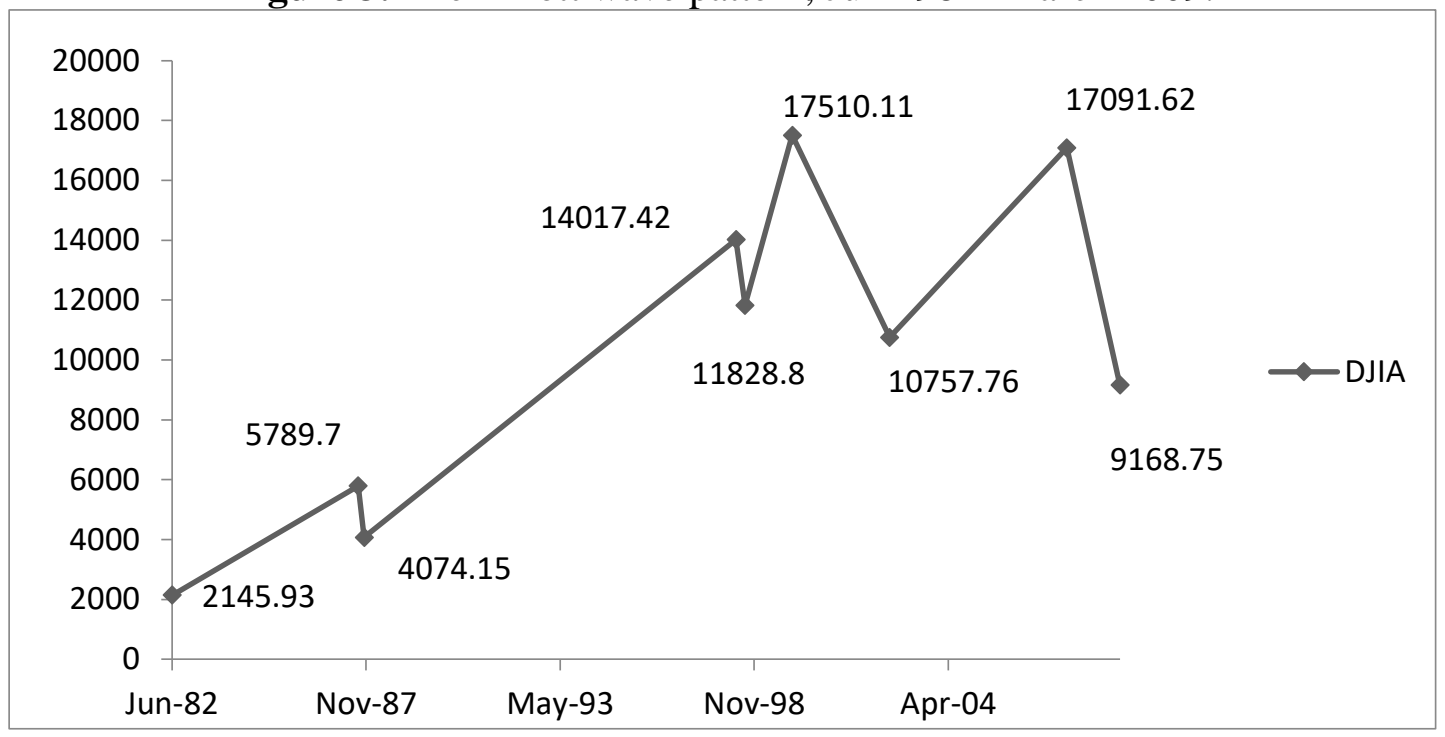

Source: http://www.macrotrends.net/1319/dow-jones-100-year-historical-chart

Further, the Elliott wave pattern, Jun 1982- March 2009. and Fibonacci ratios are shown in Figure 4. The Fibonacci ratios are included in the model (8). The model is estimated (see Table $3)$.

Figure 4. The Elliott wave pattern, Jun 1982- March 2009. and Fibonacci ratios

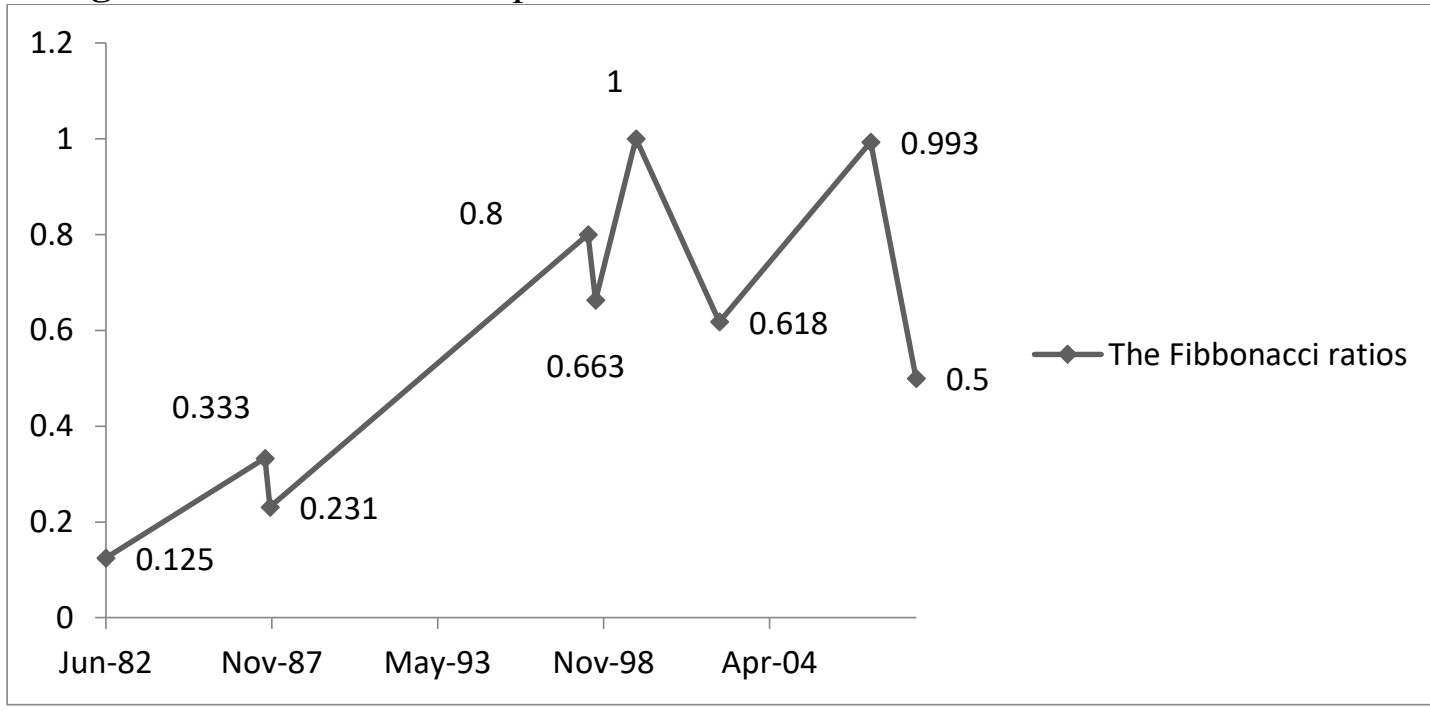

Table 3. The estimated model (8.): DJIA, Jun 1982-March 2009. (with Fibonacci ratios) $\mathrm{R}=0.63126$ Variance explained $39.849 \%$

\begin{tabular}{|l|c|c|}
\hline $\mathrm{N}=8$ & $\omega(\pi)$ & $v$ \\
\hline Estimate & $\mathbf{2 . 7 3 2 2 3 3}$ & 2.177989 \\
\hline Std.Err. & 0.554037 & 0.637897 \\
\hline $\mathrm{t}(6)$ & 4.931498 & 3.414329 \\
\hline p-level & 0.002626 & 0.014243 \\
\hline
\end{tabular}

From Jun 1982 to March 2009, the Dow Jones Industrial Average (unit measure: the Fibonacci ratios) moved from 0.125 to 0.5 . As we can see the pattern exactly follows the wave pattern 
described by Elliot Wave Theory. According to the logistic equation, for $2<\pi<3$ fluctuations converge to $\mathrm{z}=(\pi-1) / \pi$, or $(2.732233-1) / 2.732233=0.634$. According to $(7$.$) , the equilibrium$ value of the Dow Jones Industrial Average was 0.634/ (2.177989/2.732233), or 0.634 / 0.797146 or 0.79534 in the observed period. The equilibrium value of the Dow Jones Industrial Average (unit measure: the Fibonacci ratios) was $0.79534 \times 1=0.79534$ in the observed period.

\section{CONCLUSION}

This paper suggests the use of the chaotic stock price growth model in predicting the fluctuations of the stock price, or the Dow Jones Industrial Average (DJIA). The model (5) has to rely on specified parameters $\alpha, \beta, \gamma, \mu$, and initial value of the stock price, and /or the Dow Jones Industrial Average (DJIA), $p_{0}$. But even slight deviations from the values of parameters: $\alpha, \beta, \gamma, \mu$ and initial value of the stock price and/or the Dow Jones Industrial Average (DJIA), $\mathrm{p}_{0}$, show the difficulty of predicting a long-term stock price and/or DJIA.

A key hypothesis of this work is based on the idea that the coefficient $\pi=(1+\mu \alpha)$ plays a crucial role in explaining local stability of the stock price, and/or DJIA, where, $\mu$ is the adjustment coefficient; $\alpha$ is the coefficient of the stock demand function. The estimated value of the coefficient $\pi$ is $\mathbf{2 . 7 2 2 2 8 7}$. This result confirms continuous fluctuations of the stock price and/or DJIA in the period 1982-2009. The equilibrium price of the Dow Jones Industrial Average was 13923.287 in the observed period. Also, the Elliott wave pattern is observed. The Fibonacci ratios are included in the model. The equilibrium value in the modified model was 0.79534 , while the golden ratio is 0.618 .

\section{REFERENCES}

Benhabib, J., \& Day, R. H. (1981). Rational choice and erratic behaviour. Review of Economic Studies, 48, 459-471.

Benhabib, J., \& Day, R. H. (1982). Characterization of erratic dynamics in the overlapping generation model. Journal of Economic Dynamics and Control, 4, 37-55.

Day, R. H. (1982). Irregular growth cycles. American Economic Review, 72, 406-414.

Day, R. H. (1983). The emergence of chaos from classical economic growth, Quarterly Journal of Economics, 98, 200-213.

Frost A.J. \& R.P. Prechter, (2006) Elliott Wave Principle: A Key to Market Behavior. https://0104.nccdn.net/1_5/1d1/31d/37b/A.J.-Frost--Robert-Prechter---Elliott-WavePrinciple.pdf

Goodwin, R. M. (1990). Chaotic economic dynamics. Oxford: Clarendon Press.

Grandmont, J. M. (1985). On endogenous competitive business cycle. Econometrica, 53, 9941045.

Jablanović V. (2011). A Chaotic Unemployment Rate Growth Model. Hyperion International Journal of Econophysics \&New Economy, Volume 4, Issue 1, 45-51.

Jablanović, V. (2012). Budget Deficit and Chaotic Economic Growth Models. Aracne editrice S.r.l, Roma.

Jablanovic, V. (2013) Elements of Chaotic Microeconomics. Roma: Aracne editrice S.r.l.

Jablanovic, V. (2016) A Contribution to the Chaotic Economic Growth Theory. Roma: Aracne editrice S.r.l .

Li, T., \& Yorke, J. (1975) Period three implies chaos. American Mathematical Monthly, 8, 985-992.

Lidwell, W, Holden K., \& J. Butler (2010) Universal Principles of Design, Revised and Updated, Rockport Publishers, Inc. 
Lorenz, E. N. (1963). Deterministic nonperiodic flow, Journal of Atmospheric Sciences, 20, $130-141$.

Lorenz, H. W. (1993). Nonlinear dynamical economics and chaotic motion (2nd ed.). Heidelberg: Springer-Verlag.

May, R. M. (1976). Mathematical models with very complicated dynamics. Nature, 261, 459467.

Medio, A. (1993). Chaotic dynamics: Theory and applications to economics. Cambridge: Cambridge University Press.

Puu, T. (2003). Attractors, Bifurcations, and Chaos - Nonlinear Phenomena in Economics. Springer.

Zhang W.B. (2012). Discrete Dynamical Systems, Bifurcations and Chaos in Economics, Elsevier B.V. 